\title{
PCR-Assisted Contig Extension: Stepwise Strategy for Bacterial Genome Closure
}

BioTechniques 34:626-632 (March 2003)

D.M. Carraro, A.A. Camargo, A.C.M. Salim, M. Grivet ${ }^{1}$, A.T. Vasconcelos ${ }^{2}$, and A.J.G. Simpson

Ludwig Institute for Cancer Research, SP, ${ }^{1}$ CETUC - PUC, RJ, and ${ }^{2} \mathrm{LNCC} / \mathrm{MCT}$, RJ, Brazil

\section{ABSTRACT}

Finishing is rate limiting for genome projects, and improvements in the efficiency of complete genome-sequence compilation will require improved protocols for gap closure. Here we report a novel approach for extending shotgun contigs and closing gaps that we termed PCR-assisted contig extension (PACE). PACE depends on the capture of rare mismatched interactions that occur between arbitrary primers and template DNA of unknown sequence, even under highly stringent conditions, by means of elevated PCR-cycle repetition and the use of specific anchoring primers corresponding to adjacent regions of known sequence. Using PACE, we have generated extensions with an average of $1 \mathrm{~kb}$ from all contigs generated from the shotgun sequencing of a 5-Mb genome, which closed the majority of gaps with a single round of experimentation. This included the generation of multiple extensions for contigs that terminated in one of the eight copies of the rRNA operon. We calculate that the switch from shotgun sequencing to PACE should occur between 5- and 8-fold genome coverage for maximum benefit and minimum overall cost. PACE is a robust and straightforward strategy that should simplify the finishing phase of bacterial genome projects.

\section{INTRODUCTION}

Bacterial genome projects typically accrue most sequence data through high-throughput shotgun sequencing. The resulting shotgun draft is then converted into a complete representation of the organism's DNA through a more laborious completion phase. This phase is devoted mainly to the generation of additional sequence data to close gaps caused by statistical cloning fluctuations and the existence of sequences refractory to the cloning system used. The use of two or more cloning systems and libraries with different insert sizes tends to reduce the number of gaps for which no cloned DNA is available. Gaps are most frequently closed by combinatorial PCR with primers derived from contig ends (1). Nevertheless, this is ineffective for larger gaps and often generates nonspecific fragments. Alternative strategies for gap closure include subtractive hybridization (2), direct sequencing of genomic DNA $(3,4)$, and physical mapping strategies based on macro-restriction maps (5). However, all of these are technically complex and time consuming.

In the course of completing the 5$\mathrm{Mb}$ genome sequence of the free-living bacterium Chromobacterium violaceum, we used a novel approach for gap closure that we found simplified and accelerated the closure of the genome. The technique involves the generation of stepwise extensions from the ends of contigs by PCR until the closure of individual gaps is achieved. Several PCR methods have been described for the isolation of DNA segments adjacent to known sequences that could, in principle, have been exploited for such a strategy. The majority of these have been utilized for the isolation of $5^{\prime}$ flanking genomic regions of cDNA clones to clone insertion-tagged genes and to isolate the extremities of the inserts of large clones such as those in YACs (6-13). However, none of these methods have been previously evaluated as a strategy for bacterial genome closure. The majority of the relevant PCR methodologies require either processing of the template DNA before amplification or post-amplification purification and cloning steps, thus limiting their applicability within the high-throughput environment of a genome-sequencing project. However, we selected one method in which these constraints do not apply that had been originally developed for the isolation of the ends of YAC inserts (11). We have termed the stepwise application of this methodology for gap closure in bacterial genome projects PCR-assisted contig extension (PACE).

\section{MATERIALS AND METHODS}

\section{Primers}

A set of 96 primers was selected from stocks that remained from previous sequencing projects with no reference to their precise sequence, but with a preference for primers with an average of $19 \mathrm{bp}$ and a $60^{\circ} \mathrm{C}$ annealing temperature. The primers $(5 \mu \mathrm{M})$ were resuspended in water and arrayed on a 96-well plate. Pairs of outward-facing specific nested primers were designed approximately $140 \mathrm{bp}$ from contig ends and $40 \mathrm{bp}$ apart from each other. 


\section{Research Report}

\section{PCR Amplifications}

PACE was developed as a two-step PCR strategy with nested primers derived from contig ends as depicted in Figure 1. Both reactions were performed on 96-well plates in a final volume of 20 $\mu \mathrm{L}$. Reaction mixtures contained 200 $\mu \mathrm{M}$ dNTPs, $0.5 \mu \mathrm{M}$ each primer, 1.5 $\mathrm{mM} \mathrm{MgCl} 2$, Taq DNA polymerase (Invitrogen, Carlsbad, CA, USA) and appropriate buffer. In step 1, 80 ng genomic DNA and 1.5 U Taq DNA polymerase were added per reaction. In step 2 , a 1:100 dilution of the step 1 reaction products was transferred to a second 96well plate and used as a template to which was also added the same arbitrary primer used in the step 1 reaction and 1 U Taq DNA polymerase. Step 1 consisted of 60 cycles at $94^{\circ} \mathrm{C}$ for $10 \mathrm{~s}, 55^{\circ} \mathrm{C}$ for $15 \mathrm{~s}$, and $72^{\circ} \mathrm{C}$ for $2 \mathrm{~min}$, with an initial denaturation at $94^{\circ} \mathrm{C}$ for $2 \mathrm{~min}$ and a final extension at $72^{\circ} \mathrm{C}$ for $3 \mathrm{~min}$. Step 2 was identical but with an annealing temperature of $60^{\circ} \mathrm{C}$ and 40 cycles.

\section{PACE Product Analysis}

Three microliters of each reaction were loaded onto $1 \%$ agarose gels with step 1 and step 2 products in adjacent lanes. Reaction products that consisted of a single detectable band were purified with the QIAquick ${ }^{\mathrm{TM}}$ PCR Purification Kit (Qiagen, Valencia, CA, USA) and

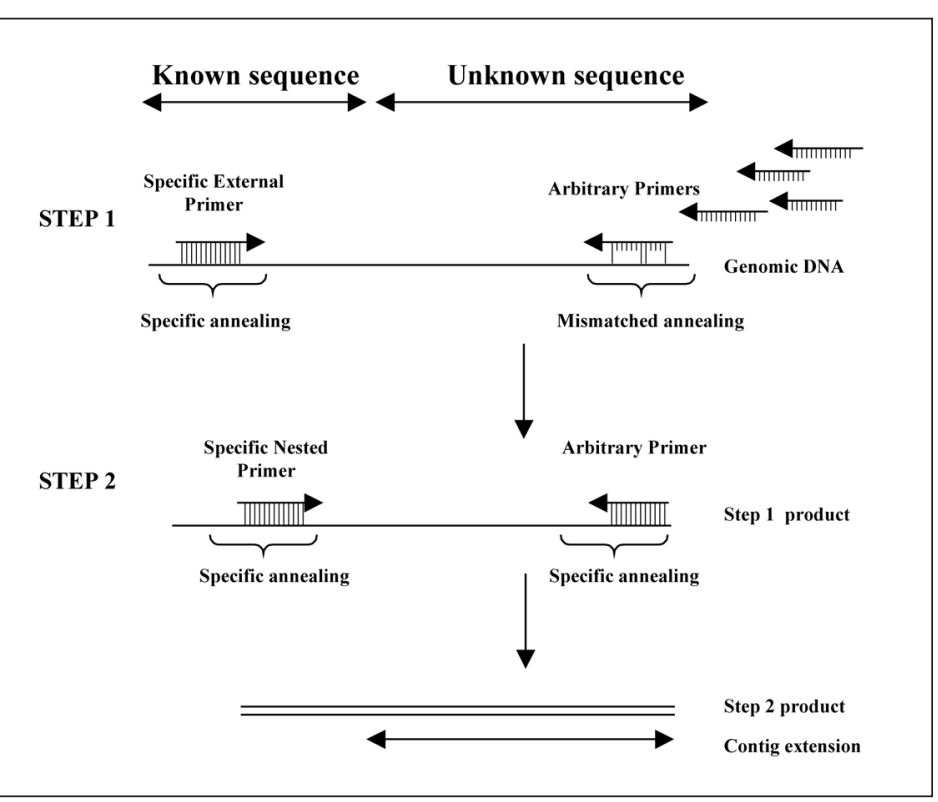

Figure 1. A schematic outline of the PACE reaction. products generated from sequenced directly with the same specific primer used for amplification on an ABI PRISM ${ }^{\circledR} 3100$ DNA sequencer (Applied Biosystems, Foster City, CA, USA). High-quality sequences with more than $300 \mathrm{bp}$ and Phred (14) quality greater than 20 were analyzed for specificity. The specificity of the PACE products was verified by BLASTN analysis against the available genome assembly. All contig extensions and gap closures were confirmed by specific PCR, followed by the direct sequencing of the PCR products.

\section{RESULTS AND DISCUSSION}

The PACE technique depends on rare mismatched primer-template interactions to be captured through elevated PCR-cycle repetition (Figure 1) and was developed in the context of the completion of the C. violaceum genome. Figure 2 shows the a typical set of PACE reactions that are all de-

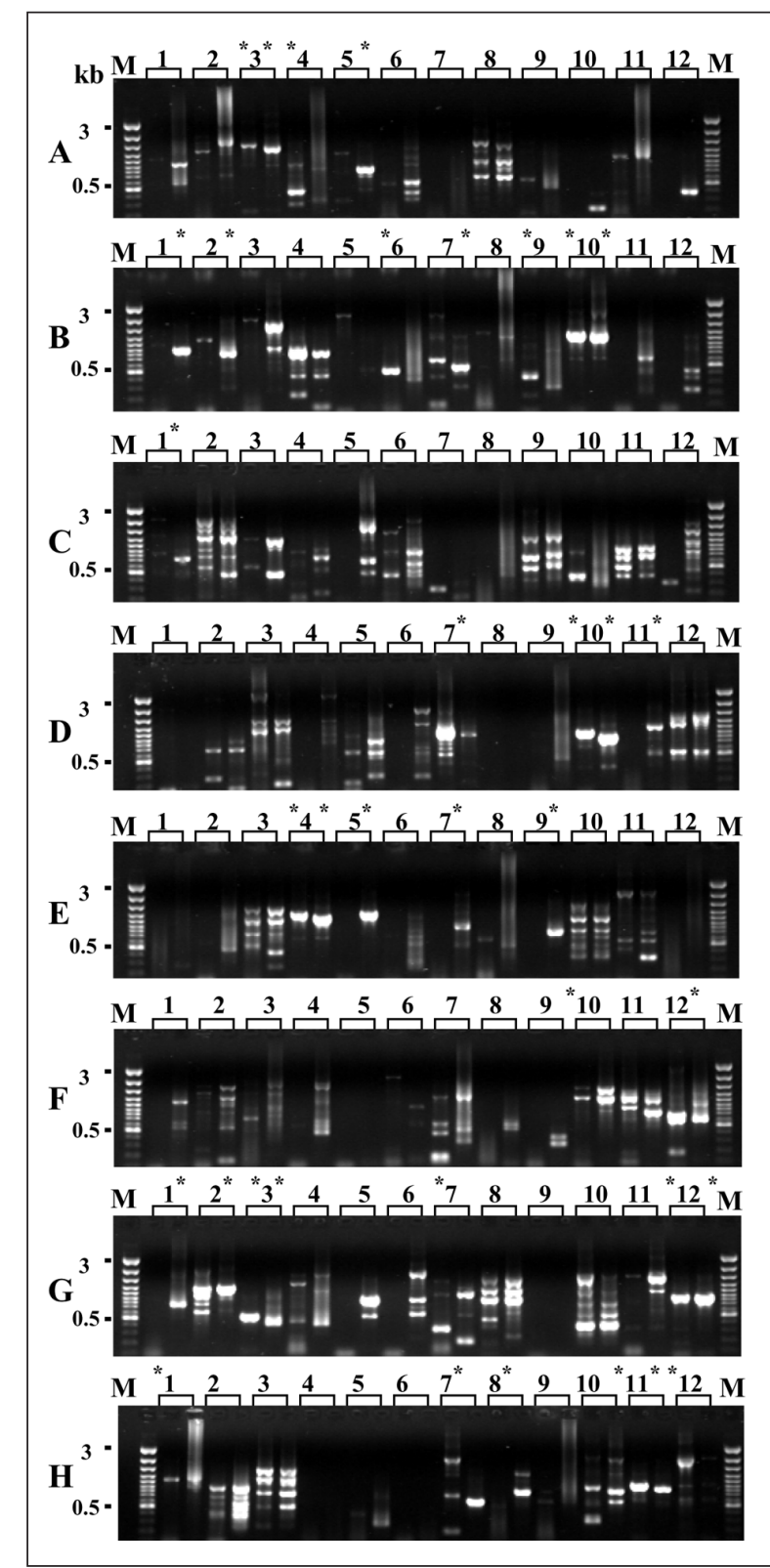

Figure 2. A $1 \%$ ethidium bromide-stained agarose gel containing PACE reaction products derived for a single $C$. violaceum contig using the same pair of nested primers for each set of step 1 and step 2 reactions. The numbers at the top and the letters $(A-H)$ on the left-hand side represent the position in the microplate. Step 1 and step 2 reactions using the same arbitrary primer have been run in adjacent lanes. The asterisks represent products selected for sequencing. M, 100-bp size marker ladder (MBI Fermentas, St. Leon-Rot, Germany). 
Table 1. Summary of Results Obtained with PACE in Extending C. violaceum Shotgun Contigs

\begin{tabular}{|c|c|c|c|c|c|c|c|c|c|}
\hline \multirow[b]{2}{*}{ Contiga } & \multicolumn{2}{|c|}{ Amplification ${ }^{b}$} & \multicolumn{2}{|c|}{ Single Products ${ }^{c}$} & \multicolumn{2}{|c|}{ Sequences $^{d}$} & \multicolumn{2}{|c|}{ Specific Productse } & \multirow{2}{*}{$\begin{array}{l}\text { Largest Single } \\
\text { Product }^{f}(\mathrm{~kb})\end{array}$} \\
\hline & step 1 & step 2 & step 1 & step 2 & step 1 & step 2 & step 1 & step 2 & \\
\hline 1 & 24 & 48 & 6 & 10 & 1 & 4 & 1 & 4 & 2.1 \\
\hline 2 & 20 & 72 & 8 & 29 & 3 & 17 & 3 & 17 & 2.5 \\
\hline 3 & 27 & 69 & 6 & 12 & 0 & 2 & 0 & 2 & 1.5 \\
\hline 4 & 34 & 76 & 12 & 27 & 0 & 4 & 0 & 4 & 2.5 \\
\hline 5 & 23 & 40 & 1 & 7 & 0 & 3 & 0 & 3 & 1.5 \\
\hline 6 & 43 & 71 & 16 & 25 & 4 & 17 & 4 & 17 & 2 \\
\hline 7 & 14 & 58 & 2 & 15 & 0 & 8 & 0 & 7 & 1.6 \\
\hline 8 & 30 & 46 & 7 & 19 & 0 & 4 & 0 & 4 & 1.7 \\
\hline 9 & 32 & 54 & 11 & 19 & 4 & 3 & 0 & 2 & 1.5 \\
\hline 10 & 32 & 72 & 15 & 21 & 2 & 7 & 0 & 7 & 1.3 \\
\hline 11 & 40 & 56 & 3 & 17 & 0 & 7 & 0 & 7 & 3 \\
\hline 12 & 33 & 45 & 10 & 22 & 3 & 8 & 3 & 8 & 1.5 \\
\hline 13 & 55 & 59 & 14 & 20 & 1 & 8 & 0 & 8 & 1.8 \\
\hline 14 & 39 & 56 & 10 & 11 & 2 & 0 & 0 & 0 & 2.1 \\
\hline 15 & 26 & 44 & 9 & 23 & 0 & 13 & 0 & 13 & 1.9 \\
\hline 16 & 46 & 55 & 6 & 14 & 0 & 4 & 0 & 4 & 1.3 \\
\hline 17 & 53 & 49 & 11 & 9 & 0 & 3 & 0 & 3 & 2.6 \\
\hline 18 & 24 & 49 & 8 & 13 & 0 & 1 & 0 & 1 & 1.5 \\
\hline 19 & 15 & 56 & 1 & 15 & 0 & 3 & 0 & 3 & 1.7 \\
\hline 20 & 36 & 53 & 6 & 11 & 0 & 8 & 0 & 7 & 1.8 \\
\hline 21 & 28 & 38 & 3 & 10 & 0 & 4 & 0 & 4 & 1.4 \\
\hline 22 & 14 & 34 & 6 & 17 & 1 & 1 & 0 & 1 & 3 \\
\hline Total & 688 & 1200 & 171 & 366 & 21 & 129 & 11 & 126 & \\
\hline \multicolumn{10}{|c|}{$\begin{array}{l}\text { aContig, number of contig ends. } \\
\text { bAmplification, number of reaction } \\
\text { cSingle product, number of reactic } \\
\text { dSequences, number of sequence } \\
\text { eSpecific products, number of spe }\end{array}$} \\
\hline
\end{tabular}

classified as authentic contig extensions when they matched with the extremity of the contig from which the pair of specific nested primers was derived. Of the 43 single fragments, 37 were selected for sequencing based on their size and intensity, and 21 generated high-quality sequence (300 bp with Phred $>20)$. All high-quality sequences were shown to be specific to the expected contig.

PACE was used to extend 22 contig ends from the $C$. violaceum genome. Table 1 summarizes the results. From the 22 sets of PACE reactions, a total of 1888 individual reactions resulted in visible products that ranged in size from 0.4 to $3 \mathrm{~kb}$, with an average size of $1 \mathrm{~kb}$. A total of 688 products were obtained from step 1 reactions, with an average of 31.3 products per set of reactions, and 1200 were obtained from step 2 reactions, with an average of 54.5 products per set of reactions. The step 2 reactions gave a higher percentage of single-band products $(35.6 \%)$ than the step 1 reactions $(24.8 \%)$.

A total of 103 single-band step 1 products and 311 step 2 products were selected for sequencing. A higher percentage of high-quality sequences were obtained from step 2 products $(41.5 \%)$ than step 1 products $(20.4 \%)$. The percentage of specific product was also much higher for the step 2 products $(97.7 \%)$ than for the step 1 products
$(52.4 \%)$. The sequencing of single PACE products allowed for the immediate closure of 15 real gaps in the $C$. violaceum genome sequence because of their relatively small size. In the remainder of the cases, additional sets of PACE reactions were undertaken until closure was achieved.

No correlation between amplification efficiency/specificity and primer length or base composition was observed (data not shown). The large number of correct PACE products per reaction suggests that the use of 32 arbitrary primers was sufficient to ensure that at least one specific fragment of $1-3 \mathrm{~kb}$ per contig. Additionally, we recommend the sequencing of only step 2 
reaction products and suggest the analysis of at least two of these for sequence confirmation.

The PACE methodology proved especially useful for extending the multicopy ribosomal operons. When we started using PACE to close the $C$. violaceum genome assembly, seven apparently identical copies of rRNA operons composed of one copy of the $5 \mathrm{~S}, 23 \mathrm{~S}$, and $16 \mathrm{~S}$ genes had been identified. Although seven contigs ended with $5 \mathrm{~S}$ rRNA gene sequence, only three ended with the 16S rRNA gene. We employed $\mathrm{PACE}$ to generate sequence flanking for the remainder of the $16 \mathrm{~S}$ rRNA genes using a single pair of nested primers. We generated a total of 21 step 1 products and 59 step 2 products of which 13 and 32, respectively, consisted of single DNA fragments. From these fragments, 35 high-quality sequences were obtained. Eighty-nine percent (8/9) of step 1 products and $100 \%(26 / 26)$ of step 2 products were found to be specific and constituted five different flanking regions for the 16S rRNA gene and the three already known, resulting in a total of eight rRNA operons. We were thus required to undertake a 5S rRNA gene-based PACE reaction to complete the final operon that had been discovered as a result of the 16S PACE protocol.
To address the optimal timing for the employment of PACE in the course of a genome project, we used the data derived from the $C$. violaceum genome assembly. We calculated the total number of PACE reactions needed to close the genome by dividing the uncovered fraction of the genome into base pairs divided by the expected average size of a PACE extension $(1 \mathrm{~kb})$. We estimated that a PACE-mediated contig extension is 24.6 times more expensive than a single shotgun read, although this will vary with primer and sequence cost. This estimate was based on the use of 32 arbitrary amplifications and the sequencing of two PCR products in both directions. While estimating the cost of the PACE reactions, we took into account the synthesis of specific step 1 and step 2 primers, Taq DNA polymerase, tips, 96-well plates, purification of PCR products, and sequencing of single products. For the shotgun-sequencing cost estimate, we considered the number of reads used in the progressive assembly of the $C$. violaceum genome and a read cost of US $\$ 3.00$.

Figure 3 presents the total genomesequencing costs when applying PACE at different stages of shotgun sequencing. If it is applied at 3-fold genome coverage, then 1920 sets of PACE reactions are required for closure, thus ele-

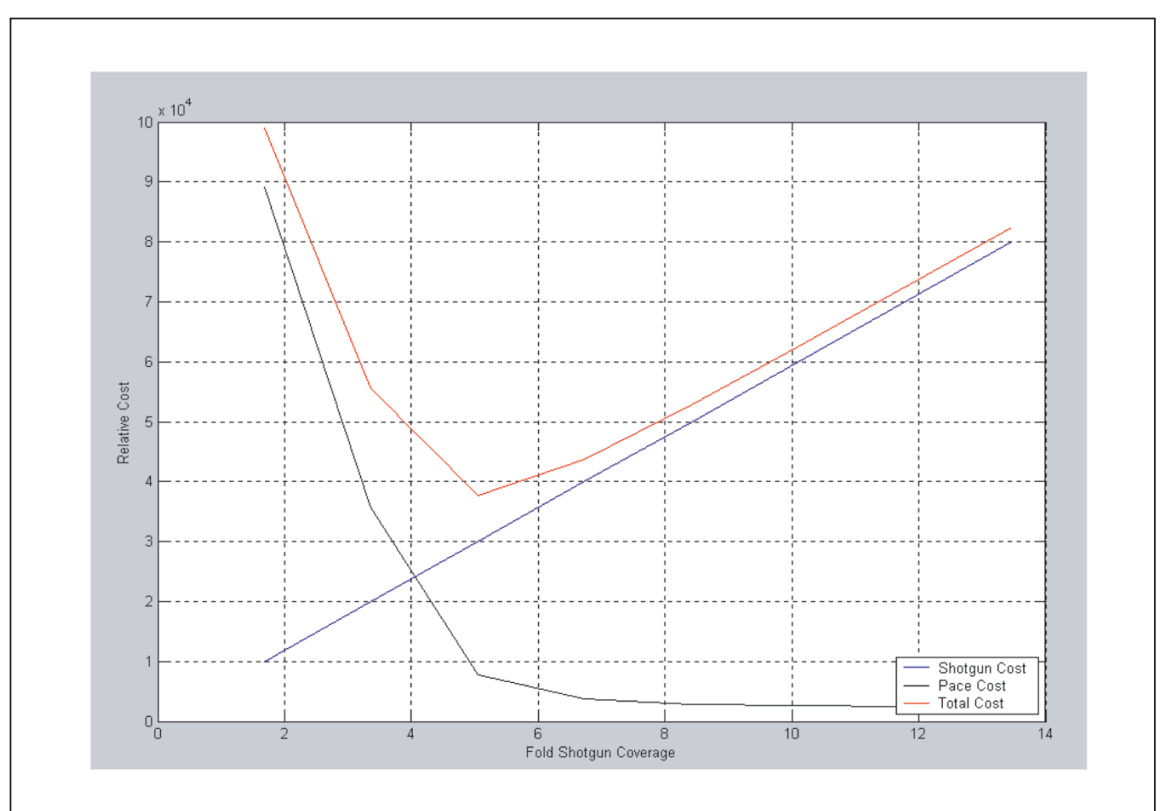

Figure 3. Relative overall cost of a complete 5-Mb genome sequence generated using PACE, following different levels of shotgun coverage. 
vating the total cost. At 6-fold coverage, the number of PACE reactions is drastically reduced (222 PACE reactions). The lowest cost for the project is obtained at approximately 5-fold genome coverage, when 345 PACE reactions are needed to close the genome. However, the continuation of shotgun sequencing until approximately 8 -fold coverage does not significantly increase the total project cost and will also considerably improve the overall quality of the sequence (Figure 3). At this point, the number of PACE reactions required is 123 of a $5-\mathrm{Mb}$ genome.

The importance of PACE is that, at a predetermined point in the shotgun sequencing, primers can be generated and contig extensions obtained with no requirement for a one-by-one analysis of the gaps. For each set of reactions, the two longest single-step 2 fragments (Table 1, column 10) can be sequenced, and the procedure can be repeated until the gap is closed. Using successive PACE reactions, it is possible to close gaps in bacterial genomes in a stepwise fashion regardless of their size. Nevertheless, the larger the gap is, the more rounds of PACE that will be required. With the availability of an in-house DNA synthesizer, this procedure could be almost entirely automated and would be expected to result in genome closure in a couple days. The approach is also applicable in other instances in which flanking sequences are required, such as in identifying the position of insertional mutation elements $(10,12,13$, 15-17). Preliminary results using the human genome DNA as a template and the current PACE format revealed a low percentage of single products ( $8.3 \%$ and $12.5 \%$ for step 1 and 2 , respectively) in comparison with the data generated for C. violaceum $[24.8 \%$ and $35.6 \%$ for step 1 and 2, respectively (Table 1)]. Of the single fragments selected for sequencing, $16.7 \%$ generated high-quality sequence; however, none of them were specific for the respective genome regions. These results suggest that the current PACE format is inappropriate for gap closure in more complex genomes, for which it will require additional modifications. Further experimentation will indicate the upper level of genome size where the current PACE protocol remains effective.

\section{ACKNOWLEDGMENTS}

The work described here was undertaken within the context of the Brazilian National Genome Program, a consortium funded in December 2000 by the Ministério de Ciência e Tecnologia (MCT) through the Conselho Nacional de Desenvolvimento Científico e Tecnológico (CNPq). We thank Dr. Juçara Parra for her general administrative coordination of this program. We are also indebted to LNCC and LICR for their administrative and technical support.

\section{REFERENCES}

1.Tettelin, H., D. Radune, S. Kasif, H. Khouri, and S.L. Salzberg. 1999. Optimized multiplex PCR: efficiently closing a whole-genome shotgun sequencing project. Genomics 15:500-507.

2.Frohme, M., A.A. Camargo, C. Czink, A.Y. Matsukuma, A.J.G. Simpson, D. Hoheisel, and S. Verjovski-Almeida. 2001. Direct gap closure in large-scale sequencing project. Genome Res. 11:901-903.

3.Heiner, C.R., K.L. Hunkapiller, S.M. Chen, J.I. Glass, and E.Y. Chen. 1998. Sequencing multimegabase-template DNA with BigDye terminator chemistry. Genome Res. 8:557561

4.Krin, E., F. Hommais, O. Soutourina, S. Noo, A. Danchin, and P. Bertin. 2001. Description and application of a rapid method for genomic DNA direct sequencing. FEMS Microbiol. Lett. 30:229-233.

5.Weinel, C., B. Tummler, H. Hilbert, K.E. Nelson, and C. Kiewitz. 2001. General method of rapid Smith/Birnstiel mapping adds for gap closure in shotgun microbial genome sequencing projects: application to Pseudomonas putida KT2440. Nucleic Acids Res. 29:E110.

6.Couch, F.J., J.M. Rommens, S.L. Neuhausen, C. Belanger, M. Dumont, K. Abel, R. Bell, S. Berry, et al. 1996. Generation of an integrated transcription map of the BRCA2 region on chromosome 13q12-q13. Genomics 36:86-99.

7.Hui, E.K., P.C. Wang, and S.J. Lo. 1995. Strategies for cloning unknown cellular flanking DNA sequences from foreign integrants. Cell Mol. Life Sci. 54:1403-1411.

8.Liu, Y.G. and R.F. Whittier. 1995. Thermal asymmetric interlaced PCR: automatable amplification and sequencing of insert end fragments from P1 and YAC clones for chromosome walking. Genomics 25:674-681.

9.Mishra, R.N., S.L. Singla-Pareek, S. Nair, S.K. Sopory, and M.K. Reddy. 2002. Directional genome walking using PCR. BioTechniques 33:831-834.

10.Spertini, D., C. Beliveau, and G. Bellemare. 1999. Screening of transgenic plants by amplification of unknown genomic DNA flanking T-DNA. BioTechniques 27:308-314.
11.Swensen, J. 1996. PCR with random primers to obtain sequence from yeast artificial chromosome insert ends or plasmids. BioTechniques 20:486-491.

12.Swensen, J., M. Hoffman, M.H. Skolnick, and S.L. Neuhausen. 1997. Identification of a $14 \mathrm{~Kb}$ deletion involving the promoter region of BRCA1 in breast cancer family. Hum. Mol. Genet. 6:1513-1517.

13.Zhou, Y.X., R.J. Newton, and J.H. Gould. 1997. A simple method for identifying plant/T-DNA junction sequences resulting from Agrobacterium-mediated DNA transformation. Plant Mol. Biol. Rep. 15:246-254.

14.Gordon, D., C. Abajian, and P. Green. 1998. Consed: a graphical tool for sequence finishing. Genome Res. 8:195-202.

15.Lukacsovich, T. and D. Yamamoto. 2001. Trap a gene and find out its function: toward functional genomics in Drosophila. J. Neurogenet. 15:147-168.

16.Mills, D.A. 2001. Mutagenesis in the post genomics era: tools for generating insertional mutations in the lactic acid bacteria. Curr. Opin. Biotechnol. 12:503-509.

17.Zheng, S.J., B. Henken, E. Sofiari, E. Jacobsen, F.A. Krens, and C. Kik. 2001. Molecular characterization of transgenic shallots (Allium cepa L.) by adaptor ligation PCR (AL-PCR) and sequencing of genomic DNA flanking T-DNA borders. Transgenic Res. 10:237-245.

Received 9 October 2002; accepted 19 December 2002.

\section{Address correspondence to:}

Dr. Andrew J.G. Simpson

Ludwig Institute for Cancer Research

605 Third Avenue

New York, NY 10158, USA

e-mail: asimpson@licr.org 